\title{
Inconsistent Safety Outcome Reporting in Randomized Clinical Trials of COVID-19 Vaccines Complicates Informed Medical Decisions
}

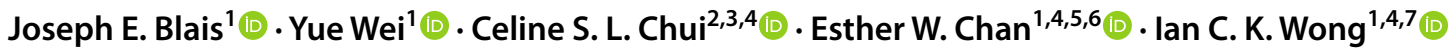

Accepted: 2 August 2021 / Published online: 17 August 2021

(c) The Author(s), under exclusive licence to Springer Nature Switzerland AG 2021

In recent months, there has been extensive reporting of interim efficacy results of several COVID-19 vaccine randomized clinical trials. Considerably less attention has been placed on the safety outcomes of these trials. Before largescale observational studies became available, individual patients and clinicians needed to make indirect comparisons between COVID-19 vaccines in regions, such as Hong Kong, where patients may choose among vaccine products. The early results of COVID-19 vaccines trials published in top medical journals [1-4] were some of the first publicly available data reporting vaccine safety outcomes, yet the reporting of these outcomes in journal articles should be more consistent, comprehensive, and transparent to allow for informed comparisons between different vaccines.

Ramasamy et al. reported local and systemic adverse reactions for Vaxzevria (COVID-19 Vaccine [ChAdOx1-S (recombinant)]; AstraZeneca AB, Stockholm, Sweden) vs the control (MenACWY vaccine) [3]. However, we note that

\section{Ian C. K. Wong}

wongick@hku.hk

1 Centre for Safe Medication Practice and Research, Department of Pharmacology and Pharmacy, LKS Faculty of Medicine, The University of Hong Kong, General Office, L02-56 2/F Laboratory Block, 21 Sassoon Road, Pokfulam, Hong Kong SAR, China

2 School of Nursing, The University of Hong Kong, Pokfulam, Hong Kong Special Administrative Region, China

3 School of Public Health, The University of Hong Kong, Pokfulam, Hong Kong Special Administrative Region, China

4 Laboratory of Data Discovery for Health, Hong Kong Science Park, Pak Shek Kok, Hong Kong Special Administrative Region, China

5 The University of Hong Kong Shenzhen Institute of Research and Innovation, Shenzhen, China

6 Department of Pharmacy, HKU-Shenzhen Hospital, Shenzhen, China

7 Research Department of Practice and Policy, UCL School of Pharmacy, London, UK the detailed adverse reaction data for the controls appear to be missing from the supplementary tables. In their studies of CoronaVac (COVID-19 Vaccine [Vero Cell], Inactivated; Sinovac Life Sciences, Beijing, China), Wu et al. and Zhang et al. define the primary safety endpoint as adverse reactions [1, 2]. The International Conference on Harmonisation defines adverse events as "Any untoward medical occurrence in a patient or clinical investigation subject administered a pharmaceutical product and which does not necessarily have to have a causal relationship with this treatment" [5]. In contrast, an adverse reaction is an event that is judged to be caused by the vaccine under study. In the published papers by $\mathrm{Wu}$ et al. and Zhang et al., there appears to be a lack of clarity in the reporting as both terms are used somewhat interchangeably. There are also discrepancies between the primary outcome of adverse reactions in the study protocols and adverse events in the statistical analysis plans.

To date, the Government of Hong Kong has purchased three COVID-19 vaccines that use three different vaccine platform technologies: Comirnaty (COVID-19 mRNA Vaccine [nucleoside-modified]; BioNTech Manufacturing $\mathrm{GmbH}$, Mainz, Germany), CoronaVac, and Vaxzevria. To emphasize the importance of consistent, clear, and transparent reporting of safety outcomes in published articles of COVID-19 vaccine randomized clinical trials, a summary of the frequency of adverse events or reactions reported in clinical trials after the first dose of these three vaccines is shown in Table 1. Published rates of adverse reactions for CoronaVac appear to be lower when compared with Comirnaty and Vaxzevria. It is not clear to us whether the investigators of CoronaVac used different definitions or different processes to ascertain whether an adverse event qualified as an adverse reaction, which could have resulted in a much lower frequency of reported adverse reactions when compared with Comirnaty and Vaxzevria, but this is difficult to ascertain from the published article. Tools created by the Brighton Collaboration [6], which establishes consistent definitions for adverse events following immunization, 
Table 1 Frequency of overall and selected vaccine adverse events $\left(\right.$ Comirnaty $\left.^{\mathrm{a}}\right)$ and adverse reactions $\left(\mathrm{CoronaVac}^{\mathrm{b}}\right.$ and Vaxzevriac ${ }^{\mathrm{c}}$ ) as reported in published COVID-19 vaccine clinical trials

\begin{tabular}{|c|c|c|c|c|c|}
\hline \multirow[t]{2}{*}{ Adverse event/reaction } & \multirow[t]{2}{*}{ Vaccine } & \multicolumn{2}{|c|}{$\begin{array}{l}18-59 / 16-55 / 18-55 \text { years } \\
\text { of age }\end{array}$} & \multicolumn{2}{|c|}{$\geq 60 / 56$ years of age } \\
\hline & & $\begin{array}{l}\text { Intervention } \\
(\%)\end{array}$ & Control (\%) & $\begin{array}{l}\text { Intervention } \\
(\%)\end{array}$ & Control (\%) \\
\hline \multirow[t]{3}{*}{ Injection-site pain } & CoronaVac & 8 & 7 & 7 & 3 \\
\hline & Comirnaty & 83 & 14 & 71 & 9 \\
\hline & Vaxzevria & 61 & NR & 29 & NR \\
\hline \multirow[t]{3}{*}{ Fatigue } & CoronaVac & 7 & 2 & 3 & 0 \\
\hline & Comirnaty & 47 & 33 & 34 & 23 \\
\hline & Vaxzevria & 76 & NR & 44 & NR \\
\hline \multirow[t]{3}{*}{ Fever } & CoronaVac & 3 & 2 & 2 & 1 \\
\hline & Comirnaty & 4 & 1 & 1 & 0 \\
\hline & Vaxzevria & 24 & NR & 0 & NR \\
\hline \multirow[t]{3}{*}{ Headache } & CoronaVac & 2 & 0 & 0 & 0 \\
\hline & Comirnaty & 42 & 34 & 25 & 18 \\
\hline & Vaxzevria & 65 & NR & 44 & NR \\
\hline \multirow[t]{3}{*}{ Overall (any adverse event) } & CoronaVac & 17 & 15 & 15 & 15 \\
\hline & Comirnaty & 27 & 12 & NR & NR \\
\hline & Vaxzevria & 98 & 74 & 85 & 48 \\
\hline
\end{tabular}

$N R$ not reported

${ }^{a}$ Outcomes are for the two-dose regimen of Comirnaty $30 \mu$ g given 21 days apart. Adverse events within 7 days of the first dose were extracted from Figure 2 of Polack et al. [4]. The overall frequency of any adverse event was only reported for the overall safety population and not stratified by age group

${ }^{b}$ Outcomes are for the two-dose regimen of CoronaVac $3 \mu \mathrm{g}$ given 28 days apart (low dose). Adverse reactions were extracted from supplementary table 3-12 for the combined phase I/II trials (Zhang et al.) [2] and from supplementary table 3-3 for the phase II trial (Wu et al.) [1] reported within 28 days of the first dose

${ }^{c}$ Outcomes are for the two-dose regimen of Vaxzevria (standard dose: $3.5-6.5 \times 10^{10}$ virus particles) given 28 days apart. Adverse reactions were solicited up to 7 days after the first dose. Specific adverse reactions were extracted from supplementary table S5-S7, while overall adverse events indicate any local or systemic adverse reaction for the priming dose (supplementary table S12) [3]. Results for the 56-69 and 70+ years of age groups were pooled have been endorsed and recommended by the World Health Organization's Global Advisory Committee on Vaccine Safety [7]. If vaccine developers used the safety templates and reported accordingly, it would greatly facilitate this area of discussion.

Moreover, we encourage the authors and their sponsors to clearly report the frequency of pooled and stratified adverse events and adverse reactions for all COVID-19 vaccine trials. Similarly, we also encourage journal reviewers and editors to carefully assess the reporting of these safety data. By providing this much needed information, patients and clinicians will be able to meet the challenge of indirectly comparing vaccine safety profiles. This safety information will enhance informed decisions about vaccine selection at the individual level, taking into consideration not only efficacy, but also the overall frequency and severity of adverse events. We note that some regulatory authorities, such as the US Food and Drug Administration and the European Medicines Agency, have made detailed COVID-19 vaccine safety data publicly available. These data, in addition to the rapidly emerging evidence from large-scale observational studies and spontaneous reports, will enhance our understanding of the comparative safety of the available COVID-19 vaccines. Observational studies are essential for assessing COVID-19 vaccine safety in populations with limited inclusion in randomized clinical trials such as very old and frail adults, pregnant women, and patients who are immunocompromised. To better inform the public, clinicians, and policy makers about the real-world comparative safety of COVID-19 vaccines, the Department of Health of Hong Kong has commissioned the COVID-19 Vaccines Adverse Events Response \& Evaluation (CARE) Programme: a comprehensive real-time surveillance study that aims to closely monitor the known and potential adverse events following immunization with COVID-19 vaccines in Hong Kong [8].

\section{Declarations}

Funding This review is funded by a Research Grant from the Food and Health Bureau, The Government of the Hong Kong Special Administrative Region (Ref. No. COVID19F01). The CARE Programme is a 
collaboration between the Hong Kong Department of Health and the Hospital Authority.

Conflict of interest The authors have no conflicts of interest to disclose.

Ethics approval Not applicable.

Consent to participate Not applicable.

Consent for publication Not applicable.

Availability of data and material Data sharing is not applicable to this article as no datasets were generated or analyzed during the current study.

Code availability Not applicable.

Author contributions JEB: literature search (English), writing (original draft and editing), conceptualization, and data extraction. YW: literature search (Chinese), writing (original draft and editing), data extraction, and data validation. CSLC: writing (editing), comments, and revision. EWC: conceptualization, writing (original draft and editing), and supervision. ICKW: comments and revision and supervision. All authors have read and approved the final version of this article.

\section{References}

1. Wu Z, Hu Y, Xu M, et al. Safety, tolerability, and immunogenicity of an inactivated SARS-CoV-2 vaccine (CoronaVac) in healthy adults aged 60 years and older: a randomised, double-blind, placebo-controlled, phase 1/2 clinical trial. Lancet Infect Dis. 2021;21(6):803-12. https://doi.org/10.1016/S1473-3099(20) 30843-4.

2. Zhang Y, Zeng G, Pan H, et al. Safety, tolerability, and immunogenicity of an inactivated SARS-CoV-2 vaccine in healthy adults aged 18-59 years: a randomised, double-blind, placebo-controlled, phase 1/2 clinical trial. Lancet Infect Dis. 2021;21(2):18192. https://doi.org/10.1016/S1473-3099(20)30987-7.

3. Ramasamy MN, Minassian AM, Ewer KJ, et al. Safety and immunogenicity of ChAdOx $1 \mathrm{nCoV}-19$ vaccine administered in a prime-boost regimen in young and old adults (COV002): a single-blind, randomised, controlled, phase $2 / 3$ trial. Lancet. 2020;396(10267):1979-93. https://doi.org/10.1016/S01406736(20)32466-1.

4. Polack FP, Thomas SJ, Kitchin N, et al. Safety and efficacy of the BNT162b2 mRNA Covid-19 vaccine. N Engl J Med. 2020;383(27):2603-15. https://doi.org/10.1056/NEJMoa2034577.

5. International Conference on Harmonisation. E2A clinical safety data management: definitions and standards for expedited reporting. ICH harmonised tripartite guideline: international conference on harmonisation; 1994. Available from: https://ich.org/page/effic acy-guidelines. Accessed 8 Aug 2021.

6. Bonhoeffer J, Kohl K, Chen R, et al. The Brighton Collaboration: addressing the need for standardized case definitions of adverse events following immunization (AEFI). Vaccine. 2002;21(3):298302. https://doi.org/10.1016/S0264-410X(02)00449-8.

7. World Health Organization. Covid-19 vaccines: safety surveillance manual. Geneva; 2020. Available from: https://www.who. int/publications/i/item/10665338400. Accessed 8 Aug 2021.

8. Department of Pharmacology and Pharmacy, The University of Hong Kong. COVID-19 Vaccines Adverse Events Response \& Evaluation (CARE) Programme. 2021. Available from: https:// www.hkcare.hku.hk/. Accessed 7 July 2021. 